\title{
Properties of the inverse of a noncentral Wishart matrix
}

\author{
Grant Hillier, CeMMAP and University of Southampton, and \\ Raymond Kan, University of Toronto
}

This version: April 2021

\begin{abstract}
The inverse of a noncentral Wishart matrix occurs in a variety of contexts in multivariate statistical work, including instrumental variables (IV) regression, but there has been very little work on its properties. In this paper we first provide an expression for the expectation of the inverse of a noncentral Wishart matrix, and then go on to do the same for a number of scalar-valued functions of the inverse. The main result is obtained by exploiting simple but powerful group-equivariance properties of the expectation map involved. Subsequent results exploit the consequences of other invariance properties.
\end{abstract}

\section{Introduction}

Many inference problems in multivariate analysis, and in econometrics, involve various properties of the non-central Wishart distribution. For example, all of the estimators for the parameters in structural equation models (LIML, FIML, OLS, IV, etc.) are functions of a non-central Wishart matrix, and the distribution theory for them derives directly from this fact. See, for instance, Phillips (1983), and the many references therein. Recent work on partially identified models (Phillips, (1989)) and weak instruments (e.g., Andrews and Stock (2005)) involves the same structure. In some applications, however, the relevant matrix is the inverse of a non-central Wishart matrix, rather than the matrix itself, and this makes the problem considerably more difficult. That is the motivation for the present paper.

If the rows of the $n \times m$ matrix $Z$ are independent normal with covariance matrix $\Sigma$, and $\mathbf{E}[Z]=M$, then, if $n \geq m$, the density of $W=Z^{\prime} Z$ is given by (Muirhead (1982), p.442)

$$
p d f(W ; \Sigma, \Lambda)=c_{m, n}|\Sigma|^{-\frac{n}{2}} \operatorname{etr}\left(-\frac{\Sigma^{-1}(\Lambda+W)}{2}\right)|W|^{\frac{n}{2}-\frac{m+1}{2}}{ }_{0} F_{1}\left(\frac{n}{2} ; \frac{\Sigma^{-1} \Lambda \Sigma^{-1} W}{4}\right),
$$


with $\Lambda=M^{\prime} M{ }^{1}$

$$
c_{m, n}=\frac{1}{2^{\frac{m n}{2}} \Gamma_{m}\left(\frac{n}{2}\right)},
$$

where $\operatorname{etr}(A)$ denotes $\exp (\operatorname{tr}(A)), \operatorname{tr}(A)$ is the trace of $A,|A|$ is the determinant of $A$, and

$$
\Gamma_{m}(t)=\pi^{\frac{m(m-1)}{4}} \prod_{i=1}^{m} \Gamma\left(t-\frac{i-1}{2}\right)
$$

is the multivariate gamma function, with $\Gamma(t)$ the univariate gamma function. Here and throughout we use the usual notation for the hypergeometric functions of matrix argument (see Constantine (1966), or Muirhead (1982)):

$$
{ }_{p} F_{q}\left(a_{1}, \ldots, a_{p} ; c_{1}, \ldots, c_{q} ; A\right)=\sum_{j=0}^{\infty} \frac{1}{j !} \sum_{\alpha \vdash j} \frac{\prod_{i=1}^{p}\left(a_{i}\right)_{\alpha}}{\prod_{r=1}^{q}\left(c_{r}\right)_{\alpha}} C_{\alpha}(A) .
$$

Here, $\alpha=\left(j_{1}, j_{2}, \ldots, j_{m}\right)$ is a partition of $j$ with at most $m$ parts (i.e., $j_{1} \geq j_{2} \geq \cdots \geq$ $j_{m} \geq 0$, and $\sum_{s=1}^{m} j_{s}=j$ ), this being denoted by $\alpha \vdash j$. The numerical coefficients $(c)_{a}$ are defined by

$$
(c)_{\alpha}=\prod_{s=1}^{m}\left(c-\frac{s-1}{2}\right)_{j_{s}},
$$

where $(c)_{r}=c(c+1) \cdots(c+r-1)$. Finally, $C_{\alpha}(\cdot)$ denotes the zonal polynomial in the elements of the indicated matrix corresponding to the partition $\alpha \vdash j$ (see Muirhead (1982), Chapter 7, or Macdonald (1995)). The important properties of these polynomials for our purposes are their invariance under orthogonal transformations, i.e., $C_{\alpha}\left(H A H^{\prime}\right)=C_{\alpha}(A), H \in \mathcal{O}(m)$, where $\mathcal{O}(m)$ is the group of $m \times m$ orthogonal matrices, and the integral identity (Constantine (1966), Equation (5)):

$$
\int_{\mathcal{O}(m)} C_{\alpha}\left(H A H^{\prime} B\right)(\mathrm{d} H)=\frac{C_{\alpha}(A) C_{\alpha}(B)}{C_{\alpha}\left(I_{m}\right)} .
$$

Here, $(\mathrm{d} H)$ denotes the normalized invariant (Haar) measure on $\mathcal{O}(m)$. We will also use the averaged hypergeometric functions based on this integral, and defined by:

$$
{ }_{p} F_{q}^{(m)}\left(a_{1}, \ldots, a_{p} ; c_{1}, \ldots, c_{q} ; A, B\right)=\int_{\mathcal{O}(m)}{ }_{p} F_{q}\left(a_{1}, \ldots, a_{p} ; c_{1}, \ldots, c_{q} ; H A H^{\prime} B\right)(\mathrm{d} H),
$$

see Muirhead (1982), p. 259.

We abbreviate the fact that $W$ has this density by $W \sim W_{m}(n, \Sigma, \Lambda)$, or $W \sim$ $W_{m}(n, \Sigma)$ when $\Lambda=0$. Expectations of functions of $W$ when $W$ has this density will

\footnotetext{
${ }^{1}$ The noncentrality matrix is usually defined as $\Omega=\Sigma^{-1} \Lambda$. Here we shall use the more symmetric notation. Also, $n$ in this definition need not be an integer: the expression given is a density for the positive definite symmetric matrix $W$ for any real number $n>m-1$.
} 
be denoted by expressions like $\mathbf{E}_{\Sigma, \Lambda}[\cdot]$, with $\Sigma$ omitted in the subscript when $\Sigma=I_{m}$, and $\Lambda$ omitted in the central case $\Lambda=0$. When $\Lambda=0$ and $\Sigma=I_{m}$ both subscripts are omitted. Analogous notation will also be used for other random variables.

In this paper we examine some of the properties of the inverse of such a matrix $W$, beginning with the expectation of $W^{-1}$. For the problem of evaluating the expectation of $W^{-1}$ we may assume that $\Sigma=I_{m}$, so that the density of $W$ depends only on $\Lambda$. For other functions of $W^{-1}$ dicussed later this assumption is restrictive, but the more general case of arbitrary $\Sigma$ is more complicated. So, later, we will discuss both of the cases $\Sigma=I_{m}$ and $\Sigma$ arbitrary. We first seek an expression for the mean, $\mathbf{E}_{\Lambda}\left[W^{-1}\right]$. In the central case where $W \sim W_{m}(n, \Sigma)$ the result is easily obtained (Muirhead (1982), p.97), and is:

$$
\mathbf{E}_{\Sigma}\left[W^{-1}\right]=(n-m-1)^{-1} \Sigma^{-1}
$$

In the (non-central) scalar case $(m=1)$ the result is also well-known, since $W \sim \chi_{n}^{2}(\lambda)$ (i.e., non-central chi-square with non-centrality parameter $\lambda$ ) and we have, for $n>2$, (Krishnan (1967))

$$
\mathbf{E}\left[\chi_{n}^{2}(\lambda)^{-1}\right]=(n-2)^{-1}{ }_{1} F_{1}\left(1 ; \frac{n}{2} ;-\frac{\lambda}{2}\right),
$$

where ${ }_{1} F_{1}(a, b ; z)$ is the confluent hypergeometric function. However, as far as we know, no explicit results are available for the non-central case when $m>1$, although some attempts on the problem have been made (Ullah (1994), Letac and Hassam (2008)). These known results are not explicit, but are expressed in terms of unresolved integrals and/or differential operators. ${ }^{2}$ The expressions obtained below are, in contrast, explicit, and relatively simple.

After evaluating $\mathbf{E}_{\Lambda}\left[W^{-1}\right]$ itself, we then go on to discuss the expectations of certain scalar functions of $W^{-1}$. Here we confine attention to orthogonally invariant functions (i.e., functions $f\left(W^{-1}\right)$ invariant under $\left.W \rightarrow H W H^{\prime}, H \in \mathcal{O}(m)\right)$. In particular, we evaluate the expectations of the elements of a particular basis for the vector space of such functions, the zonal polynomials. The expectations of functions such as $\left(\operatorname{tr}\left(W^{-1}\right)\right)^{k}$ and $\operatorname{tr}\left(W^{-k}\right)$ naturally have expansions in terms of expectations of zonal polynomials.

Throughout we denote by $e_{1}$ a unit vector $\left(e_{1}=(1,0, \ldots, 0)^{\prime}\right)$, whatever its dimension, which will be clear from the context. We denote by $\mathcal{P}_{m}$ the space of $m \times m$ symmetric matrices. The notation $A>0$ denotes the set of matrices $A \in \mathcal{P}_{m}$ that are positive-definite symmetric. Short proofs are given in the text, more elaborate ones in the Appendices.

\footnotetext{
${ }^{2}$ The expression given by Ullah (1994) is deceptively simple. However, the differential operator in fact needs to be applied to an infinite series of zonal polynomials in the variables involved in the differential operator (equation (61) below). More seriously, the action of the operator on zonal polynomials is completely unknown.
} 


\subsection{Application: IV regression}

In an IV regression with known reduced form covariance matrix we have (after suitable standardising transformations), ${ }^{3}$ the system

$$
\begin{aligned}
y & =X \beta+u, \\
X & =Z \Pi+U,
\end{aligned}
$$

with $y(n \times 1)$ independent of $X(n \times k)$ and $Z(n \times p)$ fixed (exogenous). The IV estimator for $\beta$ is the OLS estimator

$$
b=\left(X^{\prime} X\right)^{-1} X^{\prime} y,
$$

and, assuming standard normality for the rows of $(u, U)$, we have that, conditionally, given $X,(b-\beta) \mid X \sim N\left(0,\left(X^{\prime} X\right)^{-1}\right)$. Here, $W=X^{\prime} X \sim W_{k}\left(n, I_{k}, \Lambda\right)$, with

$$
\Lambda=\Pi^{\prime} Z^{\prime} Z \Pi \text {. }
$$

Thus, the conditional covariance matrix of $(b-\beta)$ is $W^{-1}$, and the unconditional covariance matrix its expectation. ${ }^{4}$

\subsection{A useful representation}

The IV regression example just discussed is useful for expository purposes. Thus, we introduce a vector $x$ whose conditional distribution, given $W$, is $N\left(0, W^{-1}\right)$. That is, the conditional covariance matrix of $x$, given $W$, is $\mathbf{E}_{x \mid W}\left[x x^{\prime}\right]=W^{-1}$, and its unconditional covariance matrix the object of our desire. But, confining attention to the case $\Sigma=I_{m}$,

$$
\mathbf{E}_{\Lambda}\left[W^{-1}\right]=\mathbf{E}_{\Lambda} \mathbf{E}_{x \mid W}\left[x x^{\prime}\right]=\mathbf{E}_{x, W}\left[x x^{\prime}\right]=\mathbf{E}_{\Lambda}\left[x x^{\prime}\right]
$$

\footnotetext{
${ }^{3}$ Briefly: we start from an equation involving $T \times(k+1)$ variables $(\tilde{y}, \tilde{Y})$, say. The IV equation is $\tilde{y}=\tilde{Y} \beta+u$, and in the background is a reduced form for $\tilde{Y}, \tilde{Y}=\tilde{Z} \Pi+U$, with $\tilde{Z} T \times p$. Since we are assuming a known covariance matrix for the rows of $(u, U)$, we may assume that standardising transformations have reduced this to an identity matrix. Also, if the IV equation involves other variables, say $Z_{1}$ (including a constant term), we assume these have been partialled out (i.e, $(\tilde{y}, \tilde{Y})$ are actually residuals after regression on $\left.Z_{1}\right)$.

The IV estimators are of the form $b=\left(\tilde{Y}^{\prime} P \tilde{Y}\right)^{-1} \tilde{Y}^{\prime} P \tilde{y}$, where $P$ is an idempotent of rank $n \geq k$. It therefore has the form $V\left(V^{\prime} V\right)^{-1} V^{\prime}$ for some choice of instruments $V(n \times k)$. Both $n$ and $V$ depend on the choice of estimator. Now define $X=\left(V^{\prime} V\right)^{-\frac{1}{2}} V^{\prime} \tilde{Y}, y=\left(V^{\prime} V\right)^{-\frac{1}{2}} V^{\prime} \tilde{y},(n \times k$ and $n \times 1$ respectively), and $Z=\left(V^{\prime} V\right)^{-\frac{1}{2}} V^{\prime} \tilde{Z}$. The estimator is then, as defined in the text, $b=\left(X^{\prime} X\right)^{-1} X^{\prime} y$. Further details can be found in Hillier et. al. (1984).

${ }^{4}$ The result we obtain below for this expectation, although reasonably complex, is considerably simpler than that given in Hillier et. al. (1984) for the second moment matrix of the IV estimator. That is because we are here assuming that the covariance matrix of $(u, U)$ is known (and so can be taken to be the identity). That assumption was not made in the earlier paper, and both the mean and covariance matrix of the conditional distribution of $b$, given $X$, there depend on $X$. This complicates matters considerably.
} 
where the final expectation is now with respect to the marginal density of $x$ :

$$
p d f(x ; \Lambda)=\int_{W>0} p d f(x \mid W) p d f(W ; \Lambda)(\mathrm{d} W) .
$$

Using a standard result on Laplace transforms of zonal polynomials (see Constantine (1963), equation (1), for example), the marginal density of $x$ is easily seen to be

$$
\begin{aligned}
p d f(x ; \Lambda)= & \frac{\Gamma_{m}\left(\frac{n+1}{2}\right) \operatorname{etr}\left(-\frac{\Lambda}{2}\right)}{\pi^{\frac{m}{2}} \Gamma_{m}\left(\frac{n}{2}\right)} \\
& \times\left(1+x^{\prime} x\right)^{-\frac{n+1}{2}}{ }_{1} F_{1}\left(\frac{n+1}{2} ; \frac{n}{2} ; \frac{1}{2} \Lambda\left(I_{m}+x x^{\prime}\right)^{-1}\right) .
\end{aligned}
$$

In the IV regression example, this equation (with $m=k$ and $\Lambda=\Pi^{\prime} Z^{\prime} Z \Pi$ ) is simply the unconditional density of the recentered IV estimator $b-\beta$.

We note, for later use, the fact that, when evaluating the expectation of any function of $x$ that is invariant under $x \rightarrow H x, H \in \mathcal{O}(m)$, the density (16) may be replaced by its average over $\mathcal{O}(m)$ under the transformation $x \rightarrow H x, H \in \mathcal{O}(m)$. Using (6), this is:

$$
\begin{aligned}
p d f^{(m)}(x ; \Lambda)= & \frac{\Gamma_{m}\left(\frac{n+1}{2}\right) \operatorname{etr}\left(-\frac{\Lambda}{2}\right)}{\pi^{\frac{m}{2}} \Gamma_{m}\left(\frac{n}{2}\right)} \\
& \times\left(1+x^{\prime} x\right)^{-\frac{n+1}{2}}{ }_{1} F_{1}^{(m)}\left(\frac{n+1}{2} ; \frac{n}{2} ; \frac{\Lambda}{2},\left(I_{m}+x x^{\prime}\right)^{-1}\right) .
\end{aligned}
$$

An exactly analogous argument applies, of course, to the density of $W$ itself, see equation (37) below.

Remark 1 This argument can easily be generalized to the case of an $m \times r$ matrix $X$ whose columns are independent $N\left(0, W^{-1}\right)$ vectors. See Section 4.2.2 below.

\section{Preliminaries}

In the representation in terms of $x$, note that, since the conditional mean $\mathbf{E}_{x}[x \mid W]$ is zero, this is also the unconditional mean. The required covariance matrix $\mathbf{E}_{\Lambda}\left[W^{-1}\right]=$ $\mathbf{E}_{\Lambda}\left[x x^{\prime}\right]$ will be a matrix-valued function of $\Lambda$, say $\Phi(\Lambda)$. The expectation operator $\mathbf{E}_{\Lambda}\left[W^{-1}\right]=\mathbf{E}_{\Lambda}\left[x x^{\prime}\right]$ thus defines a mapping from the space of symmetric matrices, $\mathcal{P}_{m}$, to itself. This map has the following simple but important property:

Lemma 1 The map $\Lambda \rightarrow \Phi(\Lambda): \mathcal{P}_{m} \rightarrow \mathcal{P}_{m}$ defined by $\Phi(\Lambda)=\mathbf{E}_{\Lambda}\left[W^{-1}\right]=\mathbf{E}_{\Lambda}\left[x x^{\prime}\right]$ is equivariant under the conjugate action of the orthogonal group $\mathcal{O}(m)$ on $\Lambda$. That is, for all $H \in \mathcal{O}(m)$,

$$
\Phi\left(H \Lambda H^{\prime}\right)=H \Phi(\Lambda) H^{\prime}
$$


Proof. On making the transformation $\Lambda \rightarrow H \Lambda H^{\prime}$, in the integral defining $\Phi$, we may then transform $x \rightarrow \tilde{x}=H^{\prime} x$, leaving the density of $\tilde{x}$ as was. Thus $\Phi\left(H \Lambda H^{\prime}\right)=$ $\mathbf{E}_{H \Lambda H^{\prime}}\left[x x^{\prime}\right]=\mathbf{E}_{\Lambda}\left[H \tilde{x} \tilde{x}^{\prime} H^{\prime}\right]=H \Phi(\Lambda) H^{\prime}$.

Lemma 1 has several valuable implications for our problem, gathered in the following:

Corollary 1 (i) If $\Lambda=L D L^{\prime}, L \in \mathcal{O}(m), D=\operatorname{diag}\left(\omega_{1}, \ldots, \omega_{m}\right)$, is the spectral decomposition of $\Lambda$, then

$$
\Phi(\Lambda)=\Phi\left(L D L^{\prime}\right)=L \Phi(D) L^{\prime} .
$$

(ii) If $\Lambda=\alpha I_{m}$, then $\Phi(\Lambda)=\bar{\psi}(\alpha) I_{m}$, for some scalar function $\bar{\psi}(\alpha)$ of $\alpha$.

(iii) For $D$ diagonal, $\Phi(D)$ is also diagonal.

Proof. (i) is just the equivariance property. (ii) Equivariance implies that $\Phi\left(\alpha I_{m}\right)=$ $\Phi\left(\alpha H I_{m} H^{\prime}\right)=H \Phi\left(\alpha I_{m}\right) H^{\prime}$ for all $H \in \mathcal{O}(m)$, which implies that $\Phi\left(\alpha I_{m}\right)$ is proportional to an identity matrix. (iii) If $\mathcal{T}$ is the subgroup of $\mathcal{O}(m)$ consisting of the $2^{m}$ diagonal matrices $T=\operatorname{diag}\{ \pm 1, \pm 1, \ldots, \pm 1\}$ (i.e., the group of sign changes), it is easy to see that $A \in \mathcal{P}_{m}$ is invariant under the action of $\mathcal{T}$ if and only if $A$ is diagonal. But, by equivariance, for every $T$ in this subgroup, $\Phi(D)=\Phi\left(T D T^{\prime}\right)=T \Phi(D) T^{\prime}$, which implies that $\Phi(D)$ is diagonal.

Translated into conclusions about the problem of interest, these results say: (i) only $p d f(x ; D)$, with $D$ the diagonal matrix of eigenvalues of $\Lambda$, need to be considered: $\Phi(\Lambda)=\mathbf{E}_{\Lambda}\left[x x^{\prime}\right]$ can be recovered from $\mathbf{E}_{D}\left[x x^{\prime}\right]$. (ii) The matrices $\Lambda$ and $\Phi(\Lambda)$ have common eigenvectors. (iii) When $\Lambda$ is spherical (proportional to the identity matrix), so is $\Phi(\Lambda)$, and (iv) variables with density $p d f(x ; D), D$ diagonal, are uncorrelated (though manifestly not independent). Thus, only the variances of the $x_{i}$ are needed to determine $\Phi(D)$. When $D=\alpha I_{m}, \operatorname{tr}(\Phi(D))=\mathbf{E}_{\Lambda}\left[x^{\prime} x\right]=m \bar{\psi}(\alpha)$, so in this case only the expectation $\mathbf{E}_{\Lambda}\left[x^{\prime} x\right]$ is needed.

The fact that $\Phi(D)$ is diagonal, coupled with its equivariance, means that the $m$ diagonal elements of $\Phi(D)$, together with the eigenvectors of $\Lambda$ in $L$, completely determine $\Phi(\Lambda)$. However, even more can be said about these diagonal elements, as follows. Let $P_{r}, r=2, \ldots, m$, denote the permutation (transposition) matrix that interchanges the first and $r$-th elements of a vector in $\mathbb{R}^{m}$. Note that the $P_{r}$ are elements of $\mathcal{O}(m)$. We have:

Lemma 2 Let $\psi_{i}(D), i=1, \ldots, m$ denote the diagonal elements of $\Psi=\Phi(D)$. Then, for each $r=2, \ldots, m, \psi_{r}(D)=\psi_{1}\left(P_{r} D P_{r}^{\prime}\right)$. That is, each of the $\psi_{r}(D)$ can be obtained from $\psi_{1}(D)$ by simply exchanging the roles of $\omega_{1}$ and $\omega_{r}, r=2, \ldots, m$.

Proof. By Lemma 1, $\Phi\left(P_{r} D P_{r}^{\prime}\right)=P_{r} \Phi(D) P_{r}^{\prime}$ for each $r=2, \ldots, m$. The $(1,1)$ element of the matrix on the left is $\psi_{1}\left(P_{r} D P_{r}^{\prime}\right)$. On the right, the $(1,1)$ element is $\psi_{r}(D)$, establishing the result. ${ }^{5}$

\footnotetext{
${ }^{5}$ In fact, for every permutation matrix $P_{\sigma}$, with $\sigma \in S_{m}$ (the symmetric group on $m$ objects), we have $\Phi\left(P_{\sigma} D P_{\sigma}^{\prime}\right)=P_{\sigma} \Phi(D) P_{\sigma}^{\prime}$. This imposes more structure on $\Phi(D)$, but the result in Lemma 2 is sufficient for our purposes.
} 
This result means that we need only determine one of the elements $\psi_{i}(D)$ of $\Psi=\Phi(D)$, say $\psi_{1}(D)$, since this determines all remaining elements. We will see the properties described in Lemma 2 directly in Section 3 (Theorem 1).

\subsection{A useful lemma}

We will make use of the following result:

Lemma 3 Let $\rho$ be a partition of an integer $r>0, C_{\rho}(\cdot)$ the zonal polynomial associated with $\rho$, and $n>m-1$ a positive real number. We have

$$
\int_{\mathbb{R}^{m}}\left(1+x^{\prime} x\right)^{-\frac{n+1}{2}} C_{\rho}\left(\left(I_{m}+x x^{\prime}\right)^{-1}\right)(\mathrm{d} x)=\frac{\pi^{\frac{m}{2}} \Gamma\left(\frac{n-m+1}{2}\right)}{\Gamma\left(\frac{n+1}{2}\right)} \frac{\left(\frac{n}{2}\right)_{\rho}}{\left(\frac{n+1}{2}\right)_{\rho}} C_{\rho}\left(I_{m}\right),
$$

and

$$
\int_{0}^{\infty} q^{\frac{m}{2}-1}(1+q)^{-\frac{n+1}{2}} C_{\rho}\left(\left(I_{m}+q e_{1} e_{1}^{\prime}\right)^{-1}\right) \mathrm{d} q=\mathrm{B}\left(\frac{m}{2}, \frac{n-m+1}{2}\right) \frac{\left(\frac{n}{2}\right)_{\rho}}{\left(\frac{n+1}{2}\right)_{\rho}} C_{\rho}\left(I_{m}\right),
$$

where $\mathrm{B}(a, c)=\Gamma(a) \Gamma(c) / \Gamma(a+c)$ is the beta coefficient.

The proof is in Appendix A. Using Lemma 3, the following corollary, which will be useful later, is easily established:

Corollary 2 For $x$ with density (17) or (16), and any $s<\frac{n-m+1}{2}$,

$$
\begin{aligned}
\mathbf{E}_{\Lambda}\left[\left(1+x^{\prime} x\right)^{s}\right]= & \frac{\Gamma\left(\frac{n+1}{2}\right) \Gamma\left(\frac{n-m+1}{2}-s\right)}{\Gamma\left(\frac{n-m+1}{2}\right) \Gamma\left(\frac{n+1}{2}-s\right)} \\
& \times \operatorname{etr}\left(-\frac{\Lambda}{2}\right){ }_{2} F_{2}\left(\frac{n+1}{2}, \frac{n}{2}-s ; \frac{n}{2}, \frac{n+1}{2}-s ; \frac{\Lambda}{2}\right) .
\end{aligned}
$$

Proof. Multiply (17) by $\left(1+x^{\prime} x\right)^{s}$ and integrate using Lemma 3 with $n$ replaced by $n-2 s$. This produces (22). Since $x^{\prime} x$ is invariant under transformations $x \rightarrow H x$, $H \in \mathcal{O}(m),(22)$ also holds when $x$ has density (16).

\section{Results for $\mathbf{E}\left[W^{-1}\right]$}

\subsection{Special cases}

In the central case $(\Lambda=0)$ we have already noted the result in equation (8). In the spherical case with $\Lambda=\alpha I_{m}, \Sigma=I_{m}$, part (ii) of Corollary 1 says that we only need to find $\bar{\psi}(\alpha)$, and this can be deduced from the fact that $\operatorname{tr}(\Phi)=\mathbf{E}_{\Lambda}\left[x^{\prime} x\right]=m \bar{\psi}(\alpha)$. Thus, we need simply to evaluate $\mathbf{E}_{\Lambda}\left[x^{\prime} x\right]$ for this case. The result is easily obtained as an application of Corollary 2: 
Proposition 1 When $\Lambda=\alpha I_{m}$ we have $\Phi\left(\alpha I_{m}\right)=\bar{\psi}(\alpha) I_{m}$, with

$$
\bar{\psi}(\alpha)=\frac{1}{m}\left[\left(\frac{n-1}{n-m-1}\right) e^{-\frac{m \alpha}{2}}{ }_{2} F_{2}\left(\frac{n+1}{2}, \frac{n-2}{2} ; \frac{n}{2}, \frac{n-1}{2} ; \frac{\alpha}{2} I_{m}\right)-1\right] .
$$

Proof. Using Corollary 2, we have when $\Lambda=\alpha I_{m}$,

$$
\mathbf{E}_{\Lambda}\left[1+x^{\prime} x\right]=\left(\frac{n-1}{n-m-1}\right) e^{-\frac{m \alpha}{2}}{ }_{2} F_{2}\left(\frac{n+1}{2}, \frac{n-2}{2} ; \frac{n}{2}, \frac{n-1}{2} ; \frac{\alpha}{2} I_{m}\right) .
$$

This gives the result stated.

When $\Lambda$ is of rank $r<m$ it can easily be shown that diagonal elements of $\Phi(D)$ corresponding to zero eigenvalues of $\Lambda$ are all equal to $(n-m-1)^{-1}$. The remaining elements will follow from the main result, the proof of which applies whatever the rank of $\Lambda$.

\subsection{Main result}

According to Lemma 2 we need only consider the case $\Lambda=D$, and also need only evaluate $\psi_{1}(D)=\operatorname{Var}\left[x_{1}\right]$, the remaining terms being obtained by transpositions $(1, r)$ of the diagonal elements of $D$. We prove:

Theorem 1 If $W \sim W_{m}\left(n, I_{m}, \Lambda\right)$, and $\Lambda=L D L^{\prime}, L \in \mathcal{O}(m), D=\operatorname{diag}\left(\omega_{1}, \ldots, \omega_{m}\right)$ is the spectral decomposition of $\Lambda$, then $\mathbf{E}_{\Lambda}\left[W^{-1}\right]=\Phi(\Lambda)=L \Psi L^{\prime}$, with $\Psi=$ $\operatorname{diag}\left(\psi_{i}(D)\right)$. When $n>m+1$, the elements on the diagonal of $\Psi=\Phi(D)$ are given by

$$
\psi_{i}(D)=\frac{\operatorname{etr}\left(-\frac{D_{i}}{2}\right)}{n-m-1} \sum_{k=0}^{\infty} \frac{\left(-\frac{\omega_{i}}{2}\right)^{k}}{\left(\frac{n}{2}\right)_{k}}{ }_{2} F_{2}\left(\frac{n}{2}, \frac{n-1}{2}+k ; \frac{n-1}{2}, \frac{n}{2}+k ; \frac{D_{i}}{2}\right),
$$

$i=1, \ldots, m$, where $D_{i}$ is $D$ with its $i$-th row and column removed. The $\psi_{i}(D)$ are the eigenvalues of $\Phi(\Lambda)$, and $\Phi(\Lambda)=L \Psi L^{\prime}$ is the spectral decomposition of $\Phi(\Lambda)$.

The proof can be found in Appendix B. It is straightforward to check that this result agrees with the known result for the case $m=1$, i.e., the case $\mathbf{E}\left[\left(\chi_{n}^{2}(\omega)\right)^{-1}\right]$. As expected (because $\Phi(\Lambda)$ is the (unconditional) covariance matrix of $x$, and $W^{-1}>0$ almost surely) we have:

Corollary 3 The matrix $\Phi(\Lambda)=\mathbf{E}_{\Lambda}\left[W^{-1}\right]$ is positive definite whatever the rank of $\Lambda$. The $\psi_{i}(D)$ corresponding to zero eigenvalues of $\Lambda$ are $(n-m-1)^{-1}$.

Remark 2 When $W \sim W_{m}(n, \Sigma, \Lambda), \tilde{W}=\Sigma^{-\frac{1}{2}} W \Sigma^{-\frac{1}{2}} \sim W_{m}\left(n, I_{m}, \Sigma^{-\frac{1}{2}} \Lambda \Sigma^{-\frac{1}{2}}\right)$, Thus, for the case $W \sim W_{m}(n, \Sigma, \Lambda)$ we have

$$
\mathbf{E}_{\Sigma, \Lambda}\left[W^{-1}\right]=\Sigma^{-\frac{1}{2}} \mathbf{E}_{\Lambda_{1}}\left[\tilde{W}^{-1}\right] \Sigma^{-\frac{1}{2}}
$$

where $\Lambda_{1}=\Sigma^{-\frac{1}{2}} \Lambda \Sigma^{-\frac{1}{2}}$, with $\mathbf{E}_{\Lambda_{1}}\left[\tilde{W}^{-1}\right]$ as given in Theorem 1 with $\Lambda$ replaced by $\Lambda_{1}$. 
Remark 3 Note that for $n$ large, the term $\mu_{k}\left(D_{1}\right)$ as defined in the proof of Theorem 1 is approximately equal to $\left(\frac{n-m+1}{2}\right)_{k} /\left(\frac{n}{2}\right)_{k}$, so that

$$
\operatorname{Var}\left[x_{1}\right] \simeq \frac{1}{n-m-1} \sum_{k=0}^{\infty} \frac{\left(-\frac{\omega_{1}}{2}\right)^{k}}{\left(\frac{n}{2}\right)_{k}}=\frac{1}{n-m-1}{ }_{1} F_{1}\left(1 ; \frac{n}{2} ;-\frac{\omega_{1}}{2}\right) .
$$

Thus, for large $n$,

$$
\Psi \simeq \frac{1}{n-m-1} \operatorname{diag}\left\{{ }_{1} F_{1}\left(1, \frac{n}{2} ;-\frac{\omega_{i}}{2}\right)\right\}
$$

with diagonal elements approximately equal to

$$
\psi_{i}(D) \simeq \frac{n-2}{n-m-1} \mathbf{E}\left[\chi_{n}^{2}\left(\omega_{i}\right)^{-1}\right], \quad i=1, \ldots, m
$$

Remark 4 Theorem 1 evidently enables the evaluation of the expectation of any linear function of the elements of $W^{-1}$. For instance, for any fixed matrix $A$,

$$
\mathbf{E}_{\Lambda}\left[\operatorname{tr}\left(A W^{-1}\right)\right]=\operatorname{tr}\left(A \mathbf{E}_{\Lambda}\left[W^{-1}\right]\right)=\operatorname{tr}\left(A L \Psi L^{\prime}\right) .
$$

\subsection{Estimation of the precision matrix $\Sigma^{-1}$}

In the central case equation (8) means that $(n-m-1) W^{-1}$ is an unbiased estimator of the precision matrix $\Sigma^{-1}$. In the non-central case this is no longer the case. Instead, as noted above,

$$
\begin{aligned}
\mathbf{E}_{\Sigma, \Lambda}\left[(n-m-1) W^{-1}\right] & =(n-m-1) \Sigma^{-\frac{1}{2}} \Phi\left(\Lambda_{1}\right) \Sigma^{-\frac{1}{2}} \\
& =\Sigma^{-1}-\Sigma^{-\frac{1}{2}}\left[I_{m}-(n-m-1) \Phi\left(\Lambda_{1}\right)\right] \Sigma^{-\frac{1}{2}}
\end{aligned}
$$

To the extent that $(n-m-1) \Phi\left(\Lambda_{1}\right)$ differs from an identity matrix, the estimator is biased by the second factor in this expression. In the case $\Lambda_{1}=\alpha I_{m}$, for instance, $I_{m}-(n-m-1) \Phi\left(\Lambda_{1}\right)=(1-(n-m-1) \bar{\psi}(\alpha)) I_{m}$ and

$$
\mathbf{E}_{\Sigma, \Lambda}\left[(n-m-1) W^{-1}\right]=(n-m-1) \bar{\psi}(\alpha) \Sigma^{-1},
$$

with $\bar{\psi}(\alpha)$ as in equation (23). For $n$ large $(n-m-1) \bar{\psi}(\alpha)$ is near 1 , but the estimator is in general biased for moderate $n$ even in this simple case.

\section{Expectation of the zonal polynomials $C_{\kappa}\left(W^{-1}\right)$}

The zonal polynomials $C_{\kappa}(A), \kappa \vdash k$, form a basis for the space of orthogonally invariant homogeneous polynomials of degree $k$ in the elements of a symmetric matrix $A$ (see, for instance, Macdonald (1995)). That is, any scalar function of $W^{-1}$ that 
is homogeneous and invariant under $W \rightarrow H W H^{\prime}, H \in \mathcal{O}(m)$ can, in principle, be expressed as a linear combination of the corresponding zonal polynomials. Scalar functions of $W^{-1}$ of potential interest in this class include the following three cases: ${ }^{1}$ powers of the determinant,

$$
\delta_{k}(\Sigma, \Lambda)=\mathbf{E}_{\Sigma, \Lambda}\left[\left|W^{-1}\right|^{k}\right], \quad k=1,2, \ldots,
$$

powers of the trace,

$$
\tau_{k}(\Sigma, \Lambda)=\mathbf{E}_{\Sigma, \Lambda}\left[\left(\operatorname{tr}\left(W^{-1}\right)\right)^{k}\right], \quad k=1,2, \ldots,
$$

and the power-sums

$$
\pi_{k}(\Sigma, \Lambda)=\mathbf{E}_{\Sigma, \Lambda}\left[\operatorname{tr}\left(W^{-k}\right)\right], \quad k=1,2, \ldots
$$

Other examples include $f_{v}(W)=\prod_{i=1}^{s} \operatorname{tr}\left(W^{-i}\right)^{\nu_{i}}$, with $\nu_{1}+2 \nu_{2}+\cdots+s \nu_{s}=k$, which can be written as linear combination of the $C_{\kappa}\left(W^{-1}\right)$ for $\kappa \vdash k$. The cases $\left(\operatorname{tr}\left(W^{-1}\right)\right)^{k}=\Sigma_{\kappa \vdash k} C_{\kappa}\left(W^{-1}\right)$ and $\operatorname{tr}\left(W^{-k}\right)$ (i.e., $\tau_{k}$ and $\left.\pi_{k}\right)$ are both in this class, with $s=1$ and $v_{1}=k$ in the first case, and $s=k, v_{i}=0$ for $i=1, \ldots, k-1$, $v_{k}=1$ in the second. Hence, if the expectations of the $C_{\kappa}\left(W^{-1}\right)$ for all $\kappa \vdash k$ are known, so are the expectations of all other functions of $W^{-1}$ in this class. This fact is the motivation for this section. The computation of the functions appearing in the results in this section is discussed in Section 5 below.

For orthogonally invariant functions $f(W)$ (including the zonal polynomials) the following results hold:

Lemma 4 Let $f(W)$ satisfy $f\left(H W H^{\prime}\right)=f(W)$ for all $H \in \mathcal{O}(m)$. Then,

1. In the case $\Sigma=I_{m}, \mathbf{E}_{\Lambda}[f(W)]$ is invariant under $\Lambda \rightarrow H \Lambda H^{\prime}, H \in \mathcal{O}(m)$, and when $\Lambda=0 \mathbf{E}_{\Sigma}[f(W)]$ is invariant under $\Sigma \rightarrow H \Sigma H^{\prime}, H \in \mathcal{O}(m)$.

2. In the case of general $\Sigma \in \mathcal{P}_{m}$, and $\Lambda \neq 0, \mathbf{E}_{\Sigma, \Lambda}[f(W)]$ is invariant under the simultaneous transformations $\Lambda \rightarrow H \Lambda H^{\prime}, \Sigma \rightarrow H \Sigma H^{\prime}, H \in \mathcal{O}(m)$.

The proofs are trivial and omitted. Evidently, in the general case the expectations $\mathbf{E}_{\Sigma, \Lambda}[f(W)]$ will involve a class of functions of $(\Sigma, \Lambda)$ more general than symmetric polynomials in either argument. We will see this directly below. However, when $\Sigma=I_{m}$ the expectations of functions in the class referred to in the Lemma depend

\footnotetext{
${ }^{1}$ Of course, for $k=1$

$$
\tau_{1}(\Sigma, \Lambda)=\pi_{1}(\Sigma, \Lambda)=\mathbf{E}_{\Sigma, \Lambda}\left[\operatorname{tr}\left(W^{-1}\right)\right],
$$

and the result follows immediately from Corollary 2 (with $s=1$ ) when $\Sigma=I_{m}$ since $\mathbf{E}_{\Lambda}\left[\operatorname{tr}\left(W^{-1}\right)\right]=$ $\operatorname{tr}(\Phi(\Lambda))=\mathbf{E}_{\Lambda}\left[x^{\prime} x\right]$.
} 
only on the eigenvalues of $\Lambda$, i.e., $D$, and may be computed from the averaged density of $W$,

$$
\begin{aligned}
p d f^{(m)}(W ; \Lambda) & =\int_{\mathcal{O}(m)} p d f\left(W ; H \Lambda H^{\prime}\right)(d H) \\
& =c_{m, n} \operatorname{etr}\left(-\frac{\Lambda+W}{2}\right)|W|^{\frac{n}{2}-\frac{m+1}{2}}{ }_{0} F_{1}^{(m)}\left(\frac{n}{2} ; \frac{\Lambda}{2}, \frac{W}{2}\right)
\end{aligned}
$$

We begin with the simplest case, the top-order zonal polynomials, with $\Sigma=I_{m}$.

\subsection{Expectations of top-order zonal polynomials}

The case of top-order zonal polynomials can be dealt with by exploiting the conditioning argument explained in Section 1.2. ${ }^{6}$ For, when $x \mid W \sim N\left(0, W^{-1}\right)$, it is straightforward to see (using the moment generating function of $q=x^{\prime} x$ ) that the conditional moments of $q$ are given by

$$
\mathbf{E}_{x \mid W}\left[q^{k} \mid W\right]=2^{k}\left(\frac{1}{2}\right)_{k} C_{k}\left(W^{-1}\right),
$$

and therefore

$$
\mathbf{E}_{\Lambda}\left[C_{k}\left(W^{-1}\right)\right]=\frac{1}{2^{k}\left(\frac{1}{2}\right)_{k}} \mathbf{E}_{\Lambda}\left[q^{k}\right],
$$

with the latter expectation evaluated in the density given in equation (17).

We may evaluate the expectations $\mathbf{E}_{\Lambda}\left[(1+q)^{k}\right]$ first, using Corollary 2, then use the (binomial) expansion

$$
q^{k}=\sum_{l=0}^{k}\left(\begin{array}{l}
k \\
l
\end{array}\right)(-1)^{l}(1+q)^{k-l}
$$

to recover $\mathbf{E}_{\Lambda}\left[q^{k}\right]$. Defining $\varphi_{k}(\Lambda):=\mathbf{E}_{\Lambda}\left[(1+q)^{k}\right], \varphi_{0}=1$, we will then have

$$
\mathbf{E}_{\Lambda}\left[q^{k}\right]=\sum_{l=0}^{k}\left(\begin{array}{l}
k \\
l
\end{array}\right)(-1)^{l} \varphi_{k-l}(\Lambda) .
$$

Thus, directly from Corollary 2, we have

\footnotetext{
${ }^{6}$ The conditioning argument used here can evidently be applied to any function of $x$, say $g(x)$, which, when $x \sim N(0, \Sigma)$, has known expectation $G(\Sigma)$. In particular, for any $g(\cdot)$ that is orthogonally invariant $(g(H x)=g(x) \forall H \in \mathcal{O}(m)), g$ depends on $x$ only through $q=x^{\prime} x, g(x)=\bar{g}(q)$ (since $q=x^{\prime} x$ is a maximal invariant under this action). In such cases we will then have

$$
\mathbf{E}_{\Lambda}\left[G\left(W^{-1}\right)\right]=\mathbf{E}_{\Lambda}[\bar{g}(q)]
$$

with the expectation on the right evaluated in the density in equation (17). The usefulness of this fact depends, of course, on whether the expectation of the function $G\left(W^{-1}\right)$ is itself of interest.
} 
Theorem 2 For $W \sim W_{m}\left(n, I_{m}, \Lambda\right)$ and $k<\frac{n-m+1}{2}$, the expectation of the top-order zonal polynomial $C_{k}\left(W^{-1}\right)$ is given by

$$
\mathbf{E}_{\Lambda}\left[C_{k}\left(W^{-1}\right)\right]=\frac{1}{2^{k}\left(\frac{1}{2}\right)_{k}} \sum_{l=0}^{k}\left(\begin{array}{l}
k \\
l
\end{array}\right)(-1)^{l} \varphi_{k-l}(\Lambda),
$$

where, for $r<\frac{n-m+1}{2}$,

$$
\begin{aligned}
\varphi_{r}(\Lambda)= & \frac{\Gamma\left(\frac{n+1}{2}\right)}{\Gamma\left(\frac{n-m+1}{2}\right)} \frac{\Gamma\left(\frac{n-m+1}{2}-r\right)}{\Gamma\left(\frac{n+1}{2}-r\right)} \\
& \times \operatorname{etr}\left(-\frac{\Lambda}{2}\right){ }_{2} F_{2}\left(\frac{n}{2}-r, \frac{n+1}{2} ; \frac{n+1}{2}-r, \frac{n}{2} ; \frac{\Lambda}{2}\right) .
\end{aligned}
$$

\subsection{General zonal polynomials}

\subsubsection{The central case}

Before tackling the non-central case we record here the results for the central case, for ease of reference later. The following result for $C_{\kappa}(W)$ is standard:

$$
\mathbf{E}_{\Sigma}\left[C_{\kappa}(W)\right]=2^{k}\left(\frac{n}{2}\right)_{\kappa} C_{\kappa}(\Sigma)
$$

For $C_{\kappa}\left(W^{-1}\right)$ the result is essentially given by Constantine (1966), equation (10), and (in different notation) Khatri (1966), Lemma 4, which we repeat here for completeness: ${ }^{1}$

Lemma 5 For $Z$ a complex symmetric matrix with $\operatorname{Re}[Z]>0, T$ complex symmetric, and $t>k_{1}+\frac{m-1}{2}$,

$$
\int_{W>0} \operatorname{etr}(-Z W)|W|^{t-\frac{m+1}{2}} C_{\kappa}\left(T W^{-1}\right)(\mathrm{d} W)=\Gamma_{m}(t,-\kappa)|Z|^{-t} C_{\kappa}(T Z),
$$

where, for $\kappa=\left(k_{1}, k_{2}, \ldots, k_{m}\right) \vdash k$,

$$
\Gamma_{m}(t,-\kappa)=\pi^{\frac{m(m-1)}{4}} \prod_{i=1}^{m} \Gamma\left(t-k_{i}-\frac{m-i}{2}\right) .
$$

Hence, for $\kappa \vdash k$, with $k<\frac{n-m+1}{2}$, and $W \sim W_{m}(n, \Sigma)$, we have (taking $\left.T=I_{m}\right)$,

$$
\mathbf{E}_{\Sigma}\left[C_{\kappa}\left(W^{-1}\right)\right]=\frac{\Gamma_{m}\left(\frac{n}{2},-\kappa\right)}{2^{k} \Gamma_{m}\left(\frac{n}{2}\right)} C_{\kappa}\left(\Sigma^{-1}\right) .
$$

\footnotetext{
${ }^{1}$ The formulae (43) and (46) reflect the fact that both $C_{\kappa}(W)$ and $C_{\kappa}\left(W^{-1}\right)$ are eigenfunctions of the family of $W_{m}(n, \Sigma)$ distributions $(n \geq m)$, i.e., that $\mathbf{E}_{W}[f(W)]=\lambda_{f, n} f(\Sigma)$, with $\lambda_{f, n}$ a constant (eigenvalue) (cf. Kushner et. al. (1981), Theorem 3.6).
} 


\subsection{2 non-central case}

For the general case, the conditioning device used in the previous sub-section for the top-order polynomials is one possible route. For, from Kushner and Meisner (1984), Equation (2.12), we have an integral expression for a zonal polynomial $C_{\kappa}\left(W^{-1}\right)$ :

$$
C_{\kappa}\left(W^{-1}\right)=\frac{1}{2^{k}\left(\frac{m}{2}\right)_{\kappa}} C_{\kappa}\left(I_{m}\right) \mathbf{E}_{X \mid W}\left[\Xi_{\kappa}\left(X^{\prime} X\right)\right],
$$

where $X$ is $m \times r$ with independent columns each distributed as $N\left(0, W^{-1}\right)$. Here $r \leq m$ is the number of non-zero parts of $\kappa$, and the function $\Xi_{\kappa}(\cdot)$, for $\kappa=$ $\left(k_{1}, k_{2}, \ldots, k_{m}\right) \vdash k$, is defined, for a symmetric matrix $A$, by

$$
\Xi_{\kappa}(A)=\left|A_{1}\right|^{k_{1}-k_{2}}\left|A_{2}\right|^{k_{2}-k_{3}} \cdots\left|A_{m-1}\right|^{k_{m-1}-k_{m}}\left|A_{m}\right|^{k_{m}},
$$

where $A_{i}$ is the upper-left $i \times i$ principal submatrix of $A$ (so $A_{1}=a_{11}$, and $A_{m}=A$ ). The expectation can, in principle, therefore be evaluated, as before, in the unconditional density of $X$ by interchanging the expectation operations. In the case $r=1$ (i.e., $C_{\kappa}(\cdot)$ is the top-order polynomial) this yields the results given in the previous subsection, since $\Xi_{(k)}\left(X^{\prime} X\right)=x_{1}^{\prime} x_{1}$, where $x_{1}$ is the first column of $X$. In general, however, this approach produces an integral that seems difficult to evaluate. We therefore adopt a different, but closely related, direct approach.

\subsection{Direct approach, non-central case}

Consider first the case $\Sigma=I_{m}$. Direct computation using the averaged density for $W$ in equation (37) means that we can write

$$
\mathbf{E}_{\Lambda}\left[C_{\kappa}\left(W^{-1}\right)\right]=\operatorname{etr}\left(-\frac{\Lambda}{2}\right) \sum_{j=0}^{\infty} \frac{1}{j !} \sum_{\alpha \vdash j} c_{\kappa, \alpha} C_{\alpha}\left(\frac{\Lambda}{2}\right),
$$

with numerical coefficients

$$
c_{\kappa, \alpha}=\frac{1}{2^{j}\left(\frac{n}{2}\right)_{\alpha} C_{\alpha}\left(I_{m}\right)} \mathbf{E}\left[C_{\kappa}\left(W^{-1}\right) C_{\alpha}(W)\right] .
$$

Recall that the absence of subsripts on $\mathbf{E}[\cdot]$ means that $W \sim W_{m}\left(n, I_{m}\right)$. The problem thus reduces to the evaluation of the expectations of the products $C_{\kappa}\left(W^{-1}\right) C_{\alpha}(W)$ when $W \sim W_{m}\left(n, I_{m}\right)$. We will give an explicit expression for these coefficients.

The key to the results given below is the following simple Lemma, expressing the zonal polynomial $C_{\kappa}\left(W^{-1}\right)$ in terms of a power of the determinant of $W$, multiplied by a zonal polynomial with argument $W$ itself:

Lemma 6 For $\kappa=\left(k_{1}, \ldots, k_{m}\right) \vdash k$ and $r$ any integer $\geq k_{1}$, the zonal polynomial $C_{\kappa}\left(W^{-1}\right)$ satisfies

$$
C_{\kappa}\left(W^{-1}\right)=\left[C_{\kappa}\left(I_{m}\right) / C_{\kappa_{r}}\left(I_{m}\right)\right]|W|^{-r} C_{\kappa_{r}}(W),
$$

where $\kappa_{r}$ is given by $\kappa_{r}=\left(r-k_{m}, r-k_{m-1}, \ldots, r-k_{1}\right)$, a partition of $r m-k$. 
Constantine (1966), p.217, mentions this relationship without proof (there the partition $\kappa_{r}$ is denoted by $\kappa^{*}$ ). Takemura (1984) states the result in Lemma 2, p.54, and gives a detailed proof. Another statement and short proof may be found in Macdonald's (2013) notes. ${ }^{7}$

Lemma 6 enables a direct evaluation of the $c_{\kappa, \alpha}$, but we also require the following result on the so-called linearization of a product of zonal polynomials (Constantine (1966), Kushner (1988), Macdonald (1995)). This says that, for $\alpha \vdash j, \lambda \vdash l$, and certain coefficients $g_{\alpha, \lambda}^{\delta}$, we have

$$
C_{\alpha}(W) C_{\lambda}(W)=\sum_{\delta \vdash j+l} g_{\alpha, \lambda}^{\delta} C_{\delta}(W) .
$$

The existence of such coefficients follows from the fact that the left-hand side is a homogeneous invariant polynomial of degree $j+l$, and the zonal polynomials span the space of such functions. The coefficients $g_{\alpha, \lambda}^{\delta}$ may not all be non-zero as $\delta$ varies over the partitions of $j+l$; they can be related to the transition matrices between the various bases of symmetric functions, and are discussed further in Section 7 below.

We also use the following easy consequence of the result in equation (43) and the linearization just given:

Lemma 7 If $W \sim W_{m}(n, \Sigma), \alpha \vdash j$, and $\lambda \vdash l$,

$$
\mathbf{E}_{\Sigma}\left[C_{\alpha}(W) C_{\lambda}(W)\right]=2^{j+l} \sum_{\delta \vdash j+l} g_{\alpha, \lambda}^{\delta}\left(\frac{n}{2}\right)_{\delta} C_{\delta}(\Sigma) .
$$

Combining the previous two Lemmas, we have: ${ }^{8}$

Lemma 8 For $\alpha \vdash j$ and $\kappa=\left(k_{1}, \ldots, k_{m}\right) \vdash k$, and $r$ an integer satisfying $k_{1} \leq r<$ $\frac{n-m+1}{2}$,

$$
\begin{aligned}
\mathbf{E}_{\Sigma}\left[C_{\kappa}\left(W^{-1}\right) C_{\alpha}(W)\right]= & {\left[\frac{2^{j-k} \Gamma_{m}\left(\frac{n}{2}-r\right) C_{\kappa}\left(I_{m}\right)}{\Gamma_{m}\left(\frac{n}{2}\right) C_{\kappa_{r}}\left(I_{m}\right)}\right] } \\
& \times|\Sigma|^{-r} \sum_{\delta \vdash r m-k+j} g_{\alpha, \kappa_{r}}^{\delta}\left(\frac{n}{2}-r\right)_{\delta} C_{\delta}(\Sigma)
\end{aligned}
$$

where $\kappa_{r}=\left(r-k_{m}, r-k_{m-1}, \ldots, r-k_{1}\right) \vdash r m-k$.

\footnotetext{
${ }^{7}$ When $m=2$ the problem simplifies because, in that case, we can take $r=k$ in Lemma 6 , and observe that $\kappa_{k}=\kappa$, so that $C_{\kappa}\left(W^{-1}\right)=|W|^{-k} C_{\kappa}(W)$, generalizing the fact $\operatorname{that}\left(\operatorname{tr}\left(W^{-1}\right)\right)^{k}=$ $|W|^{-k}(\operatorname{tr}(W))^{k}$ when $m=2$.

${ }^{8}$ Note that Lemma 8 implies that, unlike its two components, the function $f_{\kappa, \alpha}(W)=$ $C_{\kappa}\left(W^{-1}\right) C_{\alpha}(W)$ is not an eigenfunction of the $W_{m}(n, \Sigma)$ distribution, i.e., (regrettably) $\mathbf{E}_{\Sigma}\left[C_{\kappa}\left(W^{-1}\right) C_{\alpha}(W)\right]$ is not a multiple of $C_{\kappa}\left(\Sigma^{-1}\right) C_{\alpha}(\Sigma)$. The latter is, instead,

$$
C_{\alpha}(\Sigma) C_{\kappa}\left(\Sigma^{-1}\right)=\frac{C_{\kappa}\left(I_{m}\right)}{C_{\kappa_{r}}\left(I_{m}\right)}|\Sigma|^{-r} \sum_{\delta \vdash r m+j-k} g_{\alpha, \kappa_{r}}^{\delta} C_{\delta}(\Sigma) .
$$
}


Remark 5 In the central case the term $C_{\alpha}(W)$ is missing and the above result becomes,

$$
\mathbf{E}_{\Sigma}\left[C_{\kappa}\left(W^{-1}\right)\right]=\frac{\Gamma_{m}\left(\frac{n}{2}-r, \kappa_{r}\right)}{2^{k} \Gamma_{m}\left(\frac{n}{2}\right)} C_{\kappa}\left(\Sigma^{-1}\right) .
$$

This agrees with the result in equation (46), because, for any $k_{1} \leq r<\frac{n-m+1}{2}$, it is easy to see that $\Gamma_{m}\left(\frac{n}{2}-r, \kappa_{r}\right)=\Gamma_{m}\left(\frac{n}{2},-\kappa\right)$.

Applying Lemma 8 with $\Sigma=I_{m}$, we have

$$
c_{\kappa, \alpha}=\frac{\Gamma_{m}\left(\frac{n}{2}-r\right) C_{\kappa}\left(I_{m}\right)}{2^{k}\left(\frac{n}{2}\right)_{\alpha} \Gamma_{m}\left(\frac{n}{2}\right) C_{\alpha}\left(I_{m}\right) C_{\kappa_{r}}\left(I_{m}\right)} \sum_{\delta \vdash r m-k+j} g_{\alpha, \kappa_{r}}^{\delta}\left(\frac{n}{2}-r\right)_{\delta} C_{\delta}\left(I_{m}\right),
$$

which produces:

Theorem 3 For $\kappa=\left(k_{1}, \ldots, k_{m}\right) \vdash k$ with $k_{1}<\frac{n-m+1}{2}$ and $W \sim W_{m}\left(n, I_{m}, \Lambda\right)$, we have

$$
\mathbf{E}_{\Lambda}\left[C_{\kappa}\left(W^{-1}\right)\right]=\operatorname{etr}\left(-\frac{\Lambda}{2}\right) \sum_{j=0}^{\infty} \frac{1}{j !} \sum_{\alpha \vdash j} c_{\kappa, \alpha} C_{\alpha}\left(\frac{\Lambda}{2}\right)
$$

with coefficients $c_{\kappa, \alpha}$ defined as in equation (55). Here, $\kappa_{r}=\left(r-k_{m}, r-k_{m-1}, \ldots, r-\right.$ $\left.k_{1}\right) \vdash r m-k$, with $k_{1} \leq r<\frac{n-m+1}{2}$, and the coefficients $g_{\alpha, \kappa_{r}}^{\delta}$ are those appearing in the linearization (52).

\subsection{General $\Sigma$}

When $\Sigma$ is not proportional to $I_{m}$, the above argument using the averaged density (37) is not available, and an alternative approach is needed. Instead we have:

$$
\mathbf{E}_{\Sigma, \Lambda}\left[C_{\kappa}\left(W^{-1}\right)\right]=\operatorname{etr}\left(-\frac{\Sigma^{-1} \Lambda}{2}\right) \sum_{j=0}^{\infty} \frac{1}{2^{2 j} j !} \sum_{\alpha \vdash j} \frac{1}{\left(\frac{n}{2}\right)_{\alpha}} \mathbf{E}_{\Sigma}\left[C_{\kappa}\left(W^{-1}\right) C_{\alpha}\left(\Sigma^{-1} \Lambda \Sigma^{-1} W\right)\right] .
$$

The expectation term on the right-hand side is, on using Lemma 6 and Davis (1979), Equation (2.6),

$$
\begin{aligned}
& \frac{c_{m, n} C_{\kappa}\left(I_{m}\right)}{c_{m, n-2 r} C_{\kappa_{r}}\left(I_{m}\right)}|\Sigma|^{-r} \mathbf{E}_{W \sim W_{m}(n-2 r, \Sigma)}\left[C_{\kappa_{r}}(W) C_{\alpha}\left(\Sigma^{-1} \Lambda \Sigma^{-1} W\right)\right] \\
= & {\left[\frac{2^{j-k} \Gamma_{m}\left(\frac{n}{2}-r\right) C_{\kappa}\left(I_{m}\right)}{\Gamma_{m}\left(\frac{n}{2}\right) C_{\kappa_{r}}\left(I_{m}\right)}\right]|\Sigma|^{-r} \sum_{\phi \in \kappa_{r} . \alpha}\left(\frac{n}{2}-r\right)_{\phi} \theta_{\phi}^{\kappa_{r}, \alpha} C_{\phi}^{\kappa_{r}, \alpha}\left(\Sigma, \Sigma^{-1} \Lambda\right), }
\end{aligned}
$$

where $\theta_{\phi}^{\kappa_{r}, \alpha}=C_{\phi}^{\kappa_{r}, \alpha}\left(I_{m}, I_{m}\right) / C_{\phi}\left(I_{m}\right)$. The somewhat elaborate notation needed here is explained in Davis (1979). The polynomials $C_{\phi}^{\lambda, \alpha}(A, B)$ are Davis' $(1980,1981)$ invariant polynomials with two matrix arguments, invariant under the simultaneous 
transformations $A \rightarrow H A H^{\prime}, B \rightarrow H B H^{\prime}, H \in \mathcal{O}(m)$. As anticipated in Lemma 4, therefore, $\mathbf{E}_{\Sigma, \Lambda}\left[C_{\kappa}\left(W^{-1}\right)\right]$ is invariant under the simultaneous transformations $\Sigma \rightarrow$ $H \Sigma H^{\prime}, \Lambda \rightarrow H \Lambda H^{\prime}, H \in \mathcal{O}(m)$. Using this result we obtain the most general expression for the expectation of $C_{\kappa}\left(W^{-1}\right):^{9}$

Theorem 4 For $\kappa \vdash k$ and any $r$ satisfying $k_{1} \leq r<\frac{n-m+1}{2}$, and $W \sim W_{m}(n, \Sigma, \Lambda)$,

$$
\begin{aligned}
\mathbf{E}_{\Sigma, \Lambda}\left[C_{\kappa}\left(W^{-1}\right)\right]= & \frac{\Gamma_{m}\left(\frac{n}{2}-r\right) C_{\kappa}\left(I_{m}\right)}{2^{k} \Gamma_{m}\left(\frac{n}{2}\right) C_{\kappa_{r}}\left(I_{m}\right)} \operatorname{etr}\left(-\frac{\Sigma^{-1} \Lambda}{2}\right)|\Sigma|^{-r} \sum_{j=0}^{\infty} \frac{1}{2^{j} j !} \\
& \times \sum_{\alpha \vdash j} \frac{1}{\left(\frac{n}{2}\right)_{\alpha}} \sum_{\phi \in \kappa_{r} . \alpha}\left(\frac{n}{2}-r\right)_{\phi} \theta_{\phi}^{\kappa_{r}, \alpha} C_{\phi}^{\kappa_{r}, \alpha}\left(\Sigma, \Sigma^{-1} \Lambda\right) .
\end{aligned}
$$

\subsection{Applications: the functions $\delta_{k}(\Sigma, \Lambda), \tau_{k}(\Sigma, \Lambda)$, and $\pi_{k}(\Sigma, \Lambda)$}

First observe that, in the case $m=1$, the three functions coincide, being just the expected value of a negative power of a non-central $\chi_{n}^{2}(\lambda)$ variate. Hence, for $m=1$ we have, for all $k<\frac{n}{2}$,

$$
\tau_{k}(\lambda)=\pi_{k}(\lambda)=\delta_{k}(\lambda)=\frac{\Gamma\left(\frac{n}{2}-k\right)}{2^{k} \Gamma\left(\frac{n}{2}\right)}{ }_{1} F_{1}\left(k ; \frac{n}{2} ;-\frac{\lambda}{2}\right) .
$$

Note, in particular, that (unlike the expression for positive powers), this involves an infinite series in $\lambda$. It follows that, for general $m$, we cannot expect to obtain finite expansions for the expectations of these functions. Indeed, the simplest example is $\delta_{k}(\Sigma, \Lambda)=\mathbf{E}_{\Sigma, \Lambda}\left[|W|^{-k}\right]$, for which the result is given in Muirhead (1982), Theorem 10.3.7, with $r$ replaced by $-k$, and is a generalization of the result just given for $m=1:$ for $k<\frac{n-m+1}{2}$,

$$
\delta_{k}(\Sigma, \Lambda)=\frac{\Gamma_{m}\left(\frac{n}{2}-k\right)}{2^{k m} \Gamma_{m}\left(\frac{n}{2}\right)}|\Sigma|^{-k}{ }_{1} F_{1}\left(k ; \frac{n}{2} ;-\frac{\Sigma^{-1} \Lambda}{2}\right) .
$$

In contrast to the case $\mathbf{E}_{\Sigma, \Lambda}\left[|W|^{k}\right]$, which is a polynomial of degree $k m$, the series expansion for $\mathbf{E}_{\Sigma, \Lambda}\left[|W|^{-k}\right]$ does not terminate. We do not need to consider this case further.

\footnotetext{
${ }^{9}$ The analogous (infinite series) result for $\mathbf{E}_{\Sigma, \Lambda}\left[C_{\kappa}(W)\right]$ is given as Corollary 4.1 in Díaz-García and Gutiérrez-Jáimez (2001). However, we have been able to show elsewhere that it is possible to obtain an expression for the expectation of $C_{\kappa}(W)$ as a finite sum of invariant polynomials in the matrices $(\Sigma, \Lambda)$. The derivation is lengthy and entails arguments that are beyond the scope of the present paper.
} 


\subsection{1 $\tau_{k}$ and $\pi_{k}$ in the central case}

Since $\left(\operatorname{tr}\left(W^{-1}\right)\right)^{k}=\sum_{\kappa \vdash k} C_{\kappa}\left(W^{-1}\right)$, we can set $r=k$ in Lemma 6 and, using Lemma 5 , obtain at once:

$$
\mathbf{E}_{\Sigma}\left[\left(\operatorname{tr}\left(W^{-1}\right)\right)^{k}\right]=\sum_{\kappa \vdash k} \frac{\Gamma_{m}\left(\frac{n}{2},-\kappa\right)}{2^{k} \Gamma_{m}\left(\frac{n}{2}\right)} C_{\kappa}\left(\Sigma^{-1}\right)
$$

The corresponding result for the power sums $\pi_{k}$ is less straightforward. However, being a homogeneous and invariant function of $W$, the power $\operatorname{sum} \operatorname{tr}\left(W^{-k}\right)$ has an expansion in terms of zonal polynomials of the form:

$$
\operatorname{tr}\left(W^{-k}\right)=\sum_{\kappa \vdash k} d_{k, \kappa} C_{\kappa}\left(W^{-1}\right)
$$

with coefficients $d_{k, \kappa}$ that, we show in Appendix C, are given by:

$$
d_{k, \kappa}=\frac{1}{\left(k_{1}\right)_{k-k_{1}}} \prod_{i=2}^{m}\left(-\frac{i-1}{2}\right)_{k_{i}} .
$$

for $\kappa=\left(k_{1}, k_{2}, \ldots, k_{m}\right) \vdash k$, and $(c)_{0}=1$ by convention. ${ }^{10}$

Given this expansion, the result in Lemma 5 can again be invoked to yield, for the central case,

$$
\mathbf{E}_{\Sigma}\left[\operatorname{tr}\left(W^{-k}\right)\right]=\frac{1}{2^{k}} \sum_{\kappa \vdash k} \frac{d_{k, \kappa} \Gamma_{m}\left(\frac{n}{2},-\kappa\right)}{\Gamma_{m}\left(\frac{n}{2}\right)} C_{\kappa}\left(\Sigma^{-1}\right) .
$$

\subsection{2 $\tau_{k}$ and $\pi_{k}$ in the non-central case}

In the non-central case, for $\tau_{k}$ we use the expansion $\left(\operatorname{tr}\left(W^{-1}\right)\right)^{k}=\sum_{\kappa \vdash k} C_{\kappa}\left(W^{-1}\right)$, and then set $r=k$ in Lemma 6 to obtain the immediate consequence:

Corollary 4 For $k<\frac{n-m+1}{2}$, and $\Sigma=I_{m}$,

$$
\mathbf{E}_{\Lambda}\left[\left(\operatorname{tr}\left(W^{-1}\right)\right)^{k}\right]=\operatorname{etr}\left(-\frac{\Lambda}{2}\right) \sum_{j=0}^{\infty} \frac{1}{j !} \sum_{\alpha \vdash j} b_{k, \alpha} C_{\alpha}\left(\frac{\Lambda}{2}\right)
$$

with coefficients

$$
b_{k, \alpha}=\left[\frac{\Gamma_{m}\left(\frac{n}{2}-k\right)}{2^{k} \Gamma_{m}\left(\frac{n}{2}\right)\left(\frac{n}{2}\right)_{\alpha} C_{\alpha}\left(I_{m}\right)}\right] \sum_{\kappa \vdash k} \frac{C_{\kappa}\left(I_{m}\right)}{C_{\kappa_{k}}\left(I_{m}\right)} \sum_{\delta \vdash k m+j-k} g_{\alpha, \kappa_{k}}^{\delta}\left(\frac{n}{2}-k\right)_{\delta} C_{\delta}\left(I_{m}\right) .
$$

\footnotetext{
${ }^{10}$ The coefficients $d_{k, \kappa}$ are the elements in the first row of the transition matrix $\mathcal{D}_{k}$, say, that maps the zonal polynomials into the power-sum symmetric functions. The matrix $\mathcal{D}_{k}$ is not known explicitly, and equation (64) does not seem to be widely known in the literature. See Section 6 and Appendix $\mathrm{C}$ for further discussion.
} 
Finally, for $\pi_{k}(\Sigma, \Lambda)=\mathbf{E}_{\Sigma, \Lambda}\left[\operatorname{tr}\left(W^{-k}\right)\right]$ we may use equation (63), and, combining this with Lemma 6 , we have the expansion

$$
\operatorname{tr}\left(W^{-k}\right)=\sum_{\kappa \vdash k} d_{k, \kappa} C_{\kappa}\left(W^{-1}\right)=|W|^{-k} \sum_{\kappa \vdash k} d_{k, \kappa} \frac{C_{\kappa}\left(I_{m}\right)}{C_{\kappa_{k}}\left(I_{m}\right)} C_{\kappa_{k}}(W) .
$$

The coefficients $d_{k, \kappa}$ are those given earlier in equation (64). Multiplying the above expansion by $C_{\alpha}(W)$ and taking the expectation using Lemma 8 produces

Proposition 2 For $\alpha \vdash j$ and $k<\frac{n-m+1}{2}$,

$$
\mathbf{E}_{\Sigma}\left[\operatorname{tr}\left(W^{-k}\right) C_{\alpha}(W)\right]=|\Sigma|^{-k} \sum_{\delta \vdash k m-k+j} f_{k, \alpha}^{\delta}\left(\frac{n}{2}-k\right)_{\delta} C_{\delta}(\Sigma),
$$

with

$$
f_{k, \alpha}^{\delta}=\frac{2^{j-k} \Gamma_{m}\left(\frac{n}{2}-k\right)}{\Gamma_{m}\left(\frac{n}{2}\right)} \sum_{\kappa \vdash k} d_{k, \kappa} g_{\alpha, \kappa_{k}}^{\delta} \frac{C_{\kappa}\left(I_{m}\right)}{C_{\kappa_{k}}\left(I_{m}\right)} .
$$

We therefore have, for the case $\Sigma=I_{m}$,

Theorem 5 For $k<\frac{n-m+1}{2}$, and $W \sim W_{m}\left(n, I_{m}, \Lambda\right)$,

$$
\mathbf{E}_{\Lambda}\left[\operatorname{tr}\left(W^{-k}\right)\right]=\operatorname{etr}\left(-\frac{\Lambda}{2}\right) \sum_{j=0}^{\infty} \frac{1}{j !} \sum_{\alpha \vdash j} v_{k, \alpha} C_{\alpha}\left(\frac{\Lambda}{2}\right)
$$

with

$$
v_{k, \alpha}=\frac{1}{2^{j}\left(\frac{n}{2}\right)_{\alpha} C_{\alpha}\left(I_{m}\right)} \sum_{\delta \vdash k m-k+j} f_{k, \alpha}^{\delta}\left(\frac{n}{2}-k\right)_{\delta} C_{\delta}\left(I_{m}\right),
$$

where $f_{k, \alpha}^{\delta}$ is defined in Proposition 2.

In the case where $\Sigma$ is not proportional to $I_{m}$ the density-averaging device is again unavailable, and we need to rely on the Davis two-matrix-argument polynomials again. The result (really a corollary of Theorem 4) is:

Theorem 6 For $k<\frac{n-m+1}{2}$, and $W \sim W_{m}(n, \Sigma, \Lambda)$,

$$
\begin{aligned}
\mathbf{E}_{\Sigma, \Lambda}\left[\operatorname{tr}\left(W^{-k}\right)\right]= & \frac{\Gamma_{m}\left(\frac{n}{2}-k\right)}{2^{k} \Gamma_{m}\left(\frac{n}{2}\right)} \operatorname{etr}\left(-\frac{\Sigma^{-1} \Lambda}{2}\right)|\Sigma|^{-k} \sum_{j=0}^{\infty} \frac{1}{2^{j} j !} \sum_{\alpha \vdash j} \sum_{\kappa \vdash k} \frac{d_{k, \kappa}}{\left(\frac{n}{2}\right)_{\alpha}} \frac{C_{\kappa}\left(I_{m}\right)}{C_{\kappa_{k}}\left(I_{m}\right)} \\
& \times \sum_{\phi \in \kappa_{k} . \alpha}\left(\frac{n}{2}-k\right)_{\phi} \theta_{\phi}^{\kappa_{k}, \alpha} C_{\phi}^{\kappa_{k}, \alpha}\left(\Sigma, \Sigma^{-1} \Lambda\right) .
\end{aligned}
$$




\section{Transition matrices and computation issues}

In order to compute $\mathbf{E}\left[W^{-1}\right]$ in Theorem 1, and expectations of the scalar functions of $W^{-1}$ discussed in Section 4, we need to compute zonal polynomials and hypergeometric functions with matrix argument. The fastest algorithms for computing the Jack polynomials (of which zonal polynomials are a special case) and hypergeometric functions with matrix arguments are those available in Koev and Edelmen (2006) and Chan et. al. (2019).

For the results in Section 4, we need to compute the transition matrices between the different bases for the space of homogeneous symmetric functions in the eigenvalues of $W$. If $w_{1}, \ldots, w_{m}$ are the eigenvalues of $W$, the three bases that we are interested in are (1) the monomial symmetric functions, (2) the power-sum symmetric functions, and (3) the zonal polynomials. For $\kappa=\left(k_{1}, \ldots, k_{m}\right) \vdash k$, the monomial symmetric function is defined as

$$
m_{\kappa}(W)=\sum_{1 \leq i_{1}<i_{2}<\cdots<i_{m} \leq m} w_{i_{1}}^{k_{1}} w_{i_{2}}^{k_{2}} \cdots w_{i_{m}}^{k_{m}}
$$

The power-sum symmetric functions are defined as

$$
p_{\kappa}(W)=p_{k_{1}} p_{k_{2}} \cdots p_{k_{m}},
$$

where $p_{i}=\sum_{j=1}^{m} w_{j}^{i}=\operatorname{tr}\left(W^{i}\right)$. For a given value of $k$, we arrange these symmetric functions in reverse lexicographical order and define the corresponding basis vectors as

$$
m^{(k)}=\left[\begin{array}{c}
m_{(k)}(W) \\
m_{(k-1,1)}(W) \\
\vdots \\
m_{\left(1^{k}\right)}(W)
\end{array}\right], p^{(k)}=\left[\begin{array}{c}
p_{(k)}(W) \\
p_{(k-1,1)}(W) \\
\vdots \\
p_{\left(1^{k}\right)}(W)
\end{array}\right], \quad C^{(k)}=\left[\begin{array}{c}
C_{(k)}(W) \\
C_{(k-1,1)}(W) \\
\vdots \\
C_{\left(1^{k}\right)}(W)
\end{array}\right] .
$$

Our particular objective is to compute the transition matrix $\mathcal{D}_{k}$ between $C^{(k)}$ and $p^{(k)}$, defined by

$$
p^{(k)}=\mathcal{D}_{k} C^{(k)} \text {. }
$$

The matrix $\mathcal{D}_{k}$ (and other analogous transition matrices) is universal, i.e., its elements do not depend on the argument matrix $W$, so, once computed, it is known. Its dimension, though - being the number of partitions of $k$ - grows rapidly with $k$. For $k \leq 5, \mathcal{D}_{k}$ is available from James (1961), and it is obviously desirable to be able to compute $\mathcal{D}_{k}$ for arbitrary $k$. This task has hitherto been regarded as prohibitively time consuming. For example, Gutiérrez et. al. (2000) spent about 8 days to compute the transition matrix that transforms $C^{(k)}$ into $m^{(k)}$ for $k=20$ using double precision. However, we have recently written programs that are vastly more efficient when com- 
pared with existing alternatives. ${ }^{11}$ Programs to implement the calculation of $\mathcal{D}_{k}$, as well as other results in the paper, are available from the authors upon request. We shall briefly outline how these are obtained.

We first compute the inverse of $\mathcal{D}_{k}$. We do this in two steps, first computing the transition matrix $\mathcal{C}_{k}$ that expands $C^{(k)}$ in terms of $m^{(k)}$, then the transition matrix $\mathcal{T}_{k}$ that expands $m^{(k)}$ in terms of $p^{(k)}$,

$$
\begin{aligned}
C^{(k)} & =\mathcal{C}_{k} m^{(k)}, \\
m^{(k)} & =\mathcal{T}_{k} p^{(k)} .
\end{aligned}
$$

We can then compute $\mathcal{D}_{k}^{-1}=\mathcal{C}_{k} \mathcal{T}_{k}$. The most efficient method for computing $\mathcal{C}_{k}$ was developed by James (1968), and implemented by Dumitriu et. al. (2007) and Jiu and Koutschan (2020). The matrix $\mathcal{T}_{k}$ can be computed using a recursive algorithm suggested by Merca (2015).

After obtaining $\mathcal{D}_{k}^{-1}$, we can take its inverse to obtain $\mathcal{D}_{k}$, but a more efficient method is to rely on the orthogonality property of the coefficients of zonal polynomials to obtain $\mathcal{D}_{k}$. Let $d_{\kappa, \lambda}$ be a typical element of $\mathcal{D}_{k}$, where $\kappa \vdash k$ and $\lambda \vdash k$. We can use James (1961) Equation (15) to show that

$$
d_{\kappa, \lambda}=\frac{d^{\lambda, \kappa} d^{k,\left(1^{k}\right)}}{d^{k, \kappa} d^{\lambda,\left(1^{k}\right)}}
$$

where $d^{\lambda, \kappa}$ is a typical element of $\mathcal{D}_{k}^{-1}$.

For the most general results obtained in the paper, the ability to compute the expressions obtained depends on the availability of transition matrices for the Davis invariant polynomials. These are matrices $\mathcal{D}_{k, l}$, say, that map a vector of polynomials $C_{\phi}^{\kappa, \lambda}(A, B)$ with $\kappa \vdash k, \lambda \vdash l, \phi \vdash k+l$, suitably ordered, into a vector of distinct products of traces like

$$
\left(\operatorname{tr}\left(A^{a_{1}} B^{b_{1}} A^{c_{1}} \cdots\right)\right)^{r_{1}}\left(\operatorname{tr}\left(A^{a_{2}} B^{b_{2}} A^{c_{2}} \cdots\right)\right)^{r_{2}} \cdots
$$

of total degree $k$ in $A, l$ in $B$. Davis (1979) gives these matrices up to degree $k+l=5$, and explains their construction in Davis (1981). We have so far not been able to extend these beyond that degree.

Finally, we need to compute the linearization coefficients $g_{\alpha, \lambda}^{\delta}$ as defined in (52). These coefficients have been extensively studied (see, e.g., Kushner (1988)), and there are combinatorial formulae available for the linearization coefficients for products of the more general class of Jack polynomials (Stanley (1989) and Macdonald (1995)), of which the zonal polynomials are a special case. However, when $\mathcal{D}_{j}^{-1}, \mathcal{D}_{l}^{-1}$ and $\mathcal{D}_{j+l}$

\footnotetext{
${ }^{11}$ For example, on an Intel i7-4790K CPU, our SageMath program can compute $\mathcal{D}_{20}$ exactly (i.e., symbolically) in less than 10 seconds. Using double precision in Matlab, the same algorithm can compute $\mathcal{D}_{20}$ in less than 1.1 seconds. The details of our algorithm will be reported elsewhere.
} 
are available, we can simply use Theorem 3.1 of Richards (1982) to compute $g_{\alpha, \lambda}^{\delta}$ with $\alpha \vdash j, \lambda \vdash l$, and $\delta \vdash j+l$ as

$$
g_{\alpha, \lambda}^{\delta}=\sum_{\mu \vdash j+l} d_{\mu \delta} \sum_{\nu \vdash j} \sum_{\substack{\rho \vdash l \\ \nu+\rho=\mu}} d^{\alpha \nu} d^{\lambda \rho} .
$$

\section{Concluding Comment}

The key to the main result of this paper is the equivariance property in Lemma 1, and it seems likely that this type of argument may well be useful in other contexts. It appears to be novel in the econometric literature. Although very technical, the results obtained for the expectations of zonal polynomials provide the basis for solving a large class of moment problems that are potentially of interest. These also seem to be new. Finally, it may be noted that all of the results presented here can easily be generalized to the complex non-central Wishart case. This distribution has important applications in physics and electronics.

\section{APPENDIX A: Proof of Lemma 3}

The functions in the integrand on the left are invariant under $x \rightarrow H x, H \in \mathcal{O}(m)$. We may therefore use the density (17) in evaluating them. Integrating over $x \in \mathbb{R}^{m}$, the coefficients of the polynomials $C_{\rho}(\Lambda)$ in the expansion of the hypergeometric function with two matrix arguments are the numerical coefficients multiplied by the expressions

$$
\frac{1}{C_{\rho}\left(I_{m}\right)} \int_{\mathbb{R}^{m}}\left(1+x^{\prime} x\right)^{-\frac{n+1}{2}} C_{\rho}\left(\left(I_{m}+x x^{\prime}\right)^{-1}\right)(\mathrm{d} x) .
$$

Since the integral must evaluate to unity, we can equate coefficients of the polynomials $C_{\rho}(\Lambda)$ in this expression and in the expansion of $\operatorname{etr}(\Lambda / 2)$ to obtain equation $(20)$. The second expression arises by transforming $x \rightarrow(q, h)$ in the first integral with $q=x^{\prime} x$ and $h=x\left(x^{\prime} x\right)^{-\frac{1}{2}}$. The integrand is constant on the unit sphere $h^{\prime} h=1$, so integrating over $h^{\prime} h=1$ produces the result. ${ }^{12}$

\section{APPENDIX B: Proof of Theorem 1}

\footnotetext{
${ }^{12}$ An alternative proof of (21) can be based on the generalized binomial expansion for zonal polynomials (Constantine (1966), Bingham (1974), Muirhead (1982), p.267).
} 
In this Appendix we use the following notation: for any $n \times k$ matrix $A$ with $\operatorname{rank}(A)=k($ so $n \geq k)$, we define $M_{A}=I_{T}-A\left(A^{\prime} A\right)^{-1} A^{\prime}$. According to Lemma 2 we need only consider the case $\Lambda=D$, i.e., $W \sim W_{m}\left(n, I_{m}, D\right)$. The conditional variance of $x_{1}$ given $W$ is the $(1,1)$ element of $W^{-1}$ :

$$
\operatorname{Var}\left[x_{1} \mid W\right]=e_{1}^{\prime} W^{-1} e_{1}=\frac{\left|W_{22}\right|}{|W|}
$$

where $W_{22}$ is a submatrix of $W$ without its first row and column. We need the expectation of this with respect to the distribution of $W$. Writing $W=Z^{\prime} Z$, we can assume that

$$
Z \sim N\left(\left(\begin{array}{c}
D^{\frac{1}{2}} \\
0
\end{array}\right), I_{n} \otimes I_{m}\right)
$$

We also write $Z=\left(z_{1}, Z_{2}\right)$, with $z_{1} n \times 1$ and $Z_{2} n \times(m-1)$. In this notation,

$$
\operatorname{Var}\left[x_{1} \mid Z\right]=\left(z_{1}^{\prime} M_{Z_{2}} z_{1}\right)^{-1} .
$$

Here, $z_{1}$ is independent of $Z_{2}$, and $z_{1} \sim N\left(\sqrt{\omega_{1}} e_{1}, I_{n}\right)$, so that, conditionally,

$$
z_{1}^{\prime} M_{Z_{2}} z_{1} \mid Z_{2} \sim \chi_{n-m+1}^{2}\left(\omega_{1} e_{1}^{\prime} M_{Z_{2}} e_{1}\right)
$$

Therefore, as in equation (9) in the text ( with $n$ replaced by $n-m+1$ ) :

$$
\mathbf{E}\left[\left(z_{1}^{\prime} M_{Z_{2}} z_{1}\right)^{-1} \mid Z_{2}\right]=\frac{1}{n-m-1}{ }_{1} F_{1}\left(1 ; \frac{n-m+1}{2} ;-\frac{\omega_{1} e_{1}^{\prime} M_{Z_{2}} e_{1}}{2}\right) .
$$

When $\omega_{1}>0$, the unconditional variance of $x_{1}$ is the expectation of this with respect to the distribution of $Z_{2}$ :

$$
\operatorname{Var}\left[x_{1}\right]=\frac{1}{n-m-1} \sum_{k=0}^{\infty} \frac{\left(-\frac{\omega_{1}}{2}\right)^{k}}{\left(\frac{n-m+1}{2}\right)_{k}} \mu_{k}\left(D_{1}\right)
$$

where

$$
\mu_{k}\left(D_{1}\right)=\mathbf{E}_{Z_{2}}\left[\left(e_{1}^{\prime} M_{Z_{2}} e_{1}\right)^{k}\right]
$$

Now let

$$
Z_{2}=\left(\begin{array}{c}
\tilde{z}_{21}^{\prime} \\
Z_{22}
\end{array}\right)
$$

with $Z_{22} \sim N\left(\left(\begin{array}{c}D_{1}^{\frac{1}{2}} \\ 0\end{array}\right), I_{n-1} \otimes I_{m-1}\right)$, and $\tilde{z}_{21} \sim N\left(0, I_{m-1}\right)$ independent of $Z_{22}$. Then, using the properties of partitioned determinants, we have

$$
e_{1}^{\prime} M_{Z_{2}} e_{1}=\frac{\left|\left(e_{1}, Z_{2}\right)^{\prime}\left(e_{1}, Z_{2}\right)\right|}{\left|Z_{2}^{\prime} Z_{2}\right|}=\frac{\left|Z_{22}^{\prime} Z_{22}\right|}{\left|Z_{22}^{\prime} Z_{22}+\tilde{z}_{21} \tilde{z}_{21}^{\prime}\right|}=\left(1+\tilde{z}_{21}^{\prime} R^{-1} \tilde{z}_{21}\right)^{-1},
$$


where $R=Z_{22}^{\prime} Z_{22} \sim W_{m-1}\left(n-1, I_{m-1}, D_{1}\right)$ is independent of $\tilde{z}_{21}$. Writing $z=R^{-\frac{1}{2}} \tilde{z}_{21}$, we have $z \mid R \sim N\left(0, R^{-1}\right)$. That is, the joint distribution properties of $(z, R)$ are exactly as above for $(x, W)$, but with $(n, m)$ replaced by $(n-1, m-1)$ and $D$ replaced by $D_{1}$. Since $e_{1}^{\prime} M_{Z_{2}} e_{1}=\left(1+z^{\prime} z\right)^{-1}$, we need to evaluate the expectations

$$
\mu_{k}\left(D_{1}\right)=\mathbf{E}_{z}\left[\left(1+z^{\prime} z\right)^{-k}\right]
$$

when $z$ has density $p d f^{(m-1)}\left(z ; D_{1}\right)$. These clearly exist for all $k \geq 0$ and we can use Corollary 2 , with $(n, m)$ replaced by $(n-1, m-1)$ and $s$ replaced by $-k$ to obtain

$$
\mu_{k}\left(D_{1}\right)=\frac{\left(\frac{n-m+1}{2}\right)_{k}}{\left(\frac{n}{2}\right)_{k}} \operatorname{etr}\left(-\frac{D_{1}}{2}\right){ }_{2} F_{2}\left(k+\frac{n-1}{2}, \frac{n}{2} ; k+\frac{n}{2}, \frac{n-1}{2} ; \frac{D_{1}}{2}\right) .
$$

Substituting this into equation (88) gives $\psi_{1}(D)$, and Lemma 2 then implies the result stated. Note that, because the hypergeometric function is symmetric in the elements of its argument, the order of the terms in $D_{i}$ is irrelevant.

\section{APPENDIX C: Other Proofs}

\section{Proof of (64)}

Consider the alternative normalization of $C_{\kappa}$, denoted by $Z_{\kappa}$ by James (1961). The expansion of $Z_{\kappa}$ in terms of power-sum symmetric polynomials is given by

$$
Z_{\kappa}=\sum_{\lambda \vdash k} \theta_{\kappa, \lambda} p_{\lambda}
$$

Writing equation (80) in terms of the $\theta_{\kappa, \lambda}$, we can write $d_{k, \kappa}$ as

$$
d_{k, \kappa}=\frac{\theta_{\kappa, k} \theta_{k,\left(1^{k}\right)}}{\theta_{k, k} \theta_{\kappa,\left(1^{k}\right)}}=\frac{\theta_{\kappa, k}}{\theta_{k, k}}
$$

because $\theta_{\kappa,\left(1^{k}\right)}=1$ for $\kappa \vdash k$ (see Macdonald (1995, p.382), Eq.10.29). An explicit expression for $\theta_{\kappa, k}$ is given in Macdonald (1995, p.383), Example 6.10.1(b). Setting $\alpha=2$ in that expression and simplifying, we obtain

$$
\theta_{\kappa, k}=2^{k-1}\left(k_{1}-1\right) ! \prod_{i=2}^{m}\left(-\frac{i-1}{2}\right)_{k_{i}} .
$$

For the special case of $\kappa=(k)$, we have

$$
\theta_{k, k}=2^{k-1}(k-1) ! .
$$


It follows that

$$
d_{k, \kappa}=\frac{1}{\left(k_{1}\right)_{k-k_{1}}} \prod_{i=2}^{m}\left(-\frac{i-1}{2}\right)_{k_{i}} .
$$

\section{Proof of Lemma 8}

For any integer $k_{1} \leq r<\frac{n-m+1}{2}$,

$$
\mathbf{E}_{\Sigma}\left[C_{\kappa}\left(W^{-1}\right) C_{\alpha}(W)\right]=\frac{c_{m, n} C_{\kappa}\left(I_{m}\right)}{c_{m, n-2 r} C_{\kappa_{r}}\left(I_{m}\right)}|\Sigma|^{-r} \mathbf{E}_{W \sim W_{m}(n-2 r, \Sigma)}\left[C_{\alpha}(W) C_{\kappa_{r}}(W)\right] .
$$

Thus, replacing $n$ with $n-2 r$ and $\lambda$ with $\kappa_{r}$ in Lemma 7 , we have

$$
\begin{aligned}
\mathbf{E}_{\Sigma}\left[C_{\kappa}\left(W^{-1}\right) C_{\alpha}(W)\right] & =2^{j+r m-k} \frac{c_{m, n} C_{\kappa}\left(I_{m}\right)}{c_{m, n-2 r} C_{\kappa_{r}}\left(I_{m}\right)}|\Sigma|^{-r} \sum_{\delta \vdash r m+j-k} g_{\alpha, \kappa_{r}}^{\delta}\left(\frac{n}{2}-r\right)_{\delta} C_{\delta}(\Sigma) \\
& =\frac{2^{j-k} \Gamma_{m}\left(\frac{n}{2}-r\right) C_{\kappa}\left(I_{m}\right)}{\Gamma_{m}\left(\frac{n}{2}\right) C_{\kappa_{r}}\left(I_{m}\right)}|\Sigma|^{-r} \sum_{\delta \vdash r m+j-k} g_{\alpha, \kappa_{r}}^{\delta}\left(\frac{n}{2}-r\right)_{\delta} C_{\delta}(\Sigma) .
\end{aligned}
$$




\section{REFERENCES}

Andrews, D.W.K. \& J.H. Stock (2005) Inference with weak instruments. In: $A d-$ vances in Economics and Econometrics, Theory and Applications: Ninth World Congress of the Econometric Society, Vol III. Blundell R., Newey W.K., and Persson T. Eds. Cambridge University Press, Cambridge.

Bingham, C. (1974) An identity involving partitional generalized binomial coefficients. Journal of Multivariate Analysis 4, 210-223.

Chan, C., V. Drensky, A. Edelman, R. Kan, \& P. Koev (2019) On computing Schur functions and series thereof. Journal of Algebraic Combinatorics, forthcoming.

Constantine, A.G. (1963) Some non-central distribution problems in multivariate analysis. Annals of Mathematical Statistics 34, 1270-1285.

Constantine, A.G. (1966) The distribution of Hotelling's generalized $T_{0}^{2}$. Annals of Mathematical Statistics 37, 215-225.

Davis, A.W. (1979) Invariant polynomials with two matrix arguments extending the zonal polynomials: Applications to multivariate distribution theory. Annals of the Institute of Statistical Mathematics 31, Part A, 465-485.

Davis, A.W. (1980) Invariant polynomials with two matrix arguments extending the zonal polynomials. In: Multivariate Analysis-V, P.R. Krishnaiah, ed. North Holland Publishing Company.

Davis, A.W. (1981) On the construction of a class of invariant polynomials in several matrices, extending the zonal polynomials. Annals of the Institute of Statistical Mathematics 33, Part A, 297-313.

Díaz-García, J., \& R. Gutiérrez-Jáimez (2001) The expected value of zonal polynomials. Sociedad de Estadística e Investigación Operativa. Test 10, 133-145.

Dumitriu, I., A. Edelman, \& G. Shuman (2007) MOPS: Multivariate orthogonal polynomials (symbolically). Journal of Symbolic Computation 42, 587-620.

Gutiérrez, R., J. Rodriguez, \& J. Sá (2000) Approximation of hypergeometric functions with matricial argument through their development in series of zonal polynomials. Electronic Transactions on Numerical Analysis 11, 121-130.

Hillier, G.H., T. Kinal, \& V.K. Srivastava (1984) On the moments of ordinary least squares and instrumental variables estimators in a general structural equation. Econometrica 52, 185-202. 
Hillier, G.H., R. Kan, \& X. Wang (2009) Computationally efficient recursions for top-order invariant polynomials with applications, Econometric Theory 25, 211242.

James, A.T. (1961) The distribution of non-central means with known covariance. Annals of Mathematical Statistics 32, 874-882.

James, A.T. (1968) Calculation of zonal polynomial coefficients by use of the LaplaceBeltrami operator. Annals of Mathematical Statistics 39, 1711-1718.

Jiu, L. \& C. Koutschan (2020) Calculation and properties of zonal polynomials. Mathematics in Computer Science 14, 623-640.

Khatri, C.G. (1966) On certain distribution problems based on positive definite quadratic functions of normal vectors. Annals of Mathematical Statistics 37, 468-479.

Koev, P. \& A. Edelman (2006) An efficient evaluation of the hypergeometric function of a matrix argument. Mathematics of Computation 75, 833-846.

Krishnan, M. (1967) The moments of a doubly non-central $t$-distribution. Journal of the American Statistical Association 62, 278-287.

Kushner, H.B. (1988) The linearization of the product of two zonal polynomials. SIAM Journal of Mathematical Analysis 19, 687-717.

Kushner, H.B., A. Lebow, \& M. Meisner (1981) Eigenfunctions of expected value operators in the Wishart distribution, II. Journal of Multivariate Analysis 11, 418-433.

Kushner, H.B. \& M. Meisner (1984) Formula for zonal polynomials. Journal of Multivariate Analysis 14, 336-347.

Letac, G. \& H. Massam (2008) The non-central Wishart as an exponential family, and its moments. Journal of Multivariate Analysis 99, 1393-1417.

Macdonald, I.G. (2013) Hypergeometric Functions, I. Notes published online at: arXiv:1309.4568v1 [math.CA].

Macdonald, I.G. (1995) Symmetric Functions and Hall Polynomials, 2nd edition. Oxford University Press.

Merca, M. (2015) Augmented monomials in terms of power sums. SpringerPlus $4: 724$.

Muirhead, R.J. (1982) Aspects of Multivariate Statistical Theory, Wiley, New York. 
Phillips, P.C.B., (1983) Exact small sample theory in the simultaneous equations model. In: Handbook of Econometrics, Vol. I, Z. Griliches and M.D. Intriligator, Eds. North Holland.

Phillips, P.C.B., (1989) Partially Identified Econometric Models. Econometric Theory, 5, 181-240.

Richards, D.St.P. (1982) Differential operators associated with zonal polynomials. I. Annals of the Institute of Statistical Mathematics 34, 111-117.

Stanley, R. (1989) Some combinatorial properties of Jack symmetric functions. Advances in Mathematics 77, 76-115.

Takemura, A. (1984) A statistical approach to zonal polynomials. Institute of Mathematical Statistics Lecture Notes Monograph Series, Volume 4, Hayward, California.

Ullah, A. (1994) On the inverse moments of the non central Wishart matrix. Parisankhyan Samikkha 1, 49-50.

Wilf, H.S. (2005) Generatingfunctionology, 3rd edition. A.K. Peters. 